\title{
Teslas Paukenschlag - eine Chance für Brandenburg
}

Es war ein Paukenschlag, als Tesla-Chef Elon Musk im November 2019 verkündete, ausgerechnet in Brandenburg eine neue Giga-Factory bauen zu wollen: 500000 Elektroautos pro Jahr - 10000 neue Arbeitsplätze - Startschuss schon 2020. Fast zu schön, um wahr zu sein, weswegen viele dem berüchtigten Visionär keinen Glauben schenken wollten. Dass die Sache jetzt zu scheitern drohte, liegt weder an unternehmerischer Großspurigkeit noch an deutscher Bürokratie oder Naturverbundenheit. Hier ziehen ausgebuffte Lobbyisten alle juristischen Register und sabotieren das bislang reibungslose Zusammenspiel zwischen Wirtschaft, Politik und Klimaschutz.

Ökonomie und Ökologie sind zwei Seiten derselben Medaille, wenn es um das Klima geht. Wollen wir den Kosten-Tsunami der fossilen Welt verkleinern, müssen wir in erneuerbare Technologien investieren. Elektromobilität wird ein zentraler Baustein für eine nachhaltige Verkehrswende sein. Strom aus Ökoenergien direkt für die Mobilität zu nutzen, ist umweltfreundlich und effizient. Batterien werden im Rahmen der Sektorkopplung als Speicher dienen. Digitalisierung und autonomes Fahren ermöglichen neue Formen individueller Mobilität. Die Zukunft gehört denen, die sie sehen. Und wer nicht mit der Zeit geht, geht mit der Zeit.

Tesla ist deutschen Herstellern weit voraus. Aber auch VW oder Porsche orientieren sich mittlerweile zu Recht um. Dass nun der kalifornische Hersteller den deutschen Herstellern im eigenen Land Konkurrenz machen will, ist gut und richtig. Konkurrenz belebt das Geschäft. Und Deutschland kann Investitionen gut gebrauchen, nicht nur um die eigene Wettbewerbsfähigkeit zu stärken, sondern auch als Signal an die Welt.

Der Konzern drückt aufs Tempo. Das Land Brandenburg reagierte - entgegen allen Vorurteilen - schnell und flexibel. Viele Vorschriften und Genehmigungsverfahren absolvierte der Konzern im Eiltempo. Kurzfristig bremste ein gemeinnütziger Verein aus Bayern rigoros die Zukunftsreise und stoppt per Eilantrag die Rodung eines Kiefernwaldes. Vorgeblich geht es um Natur- und Artenschutz. Auf den ersten Blick berechtigt: Selbstverständlich müssen deutsche Umwelt- und Naturschutz-Vorgaben eingehalten werden. Doch Tesla tut das. Der kalifornische Konzern kennt solche Gesetze aus dem eigenen Land zur Genüge. Weder verlangt er Sonderrechte, noch bekommt er sie. Schon jetzt ist klar, dass Tesla nach der Rodung entsprechende Ausgleichswälder bereitstellen wird.

Worum geht es also? Sind da übereifrige Krötenretter am Werk, denen eine Kiefernplantage wertvoller ist als ein florierendes Unternehmen? Freunde der Bäume, aber Feinde der Wirtschaft? Tatsächlich protestieren in Grünheide auch radikale Antikapitalisten, denen es weniger um gedeihende Pflanzen geht, sondern vielmehr darum, das Wachstum der Wirtschaft möglichst zu verhindern. Genau wie bei den Gegnern der Energiewende dient einigen Verbänden die Natur lediglich als Feigenblatt ihrer wahren politischen Interessen. Typisch radikale Ökos eben?

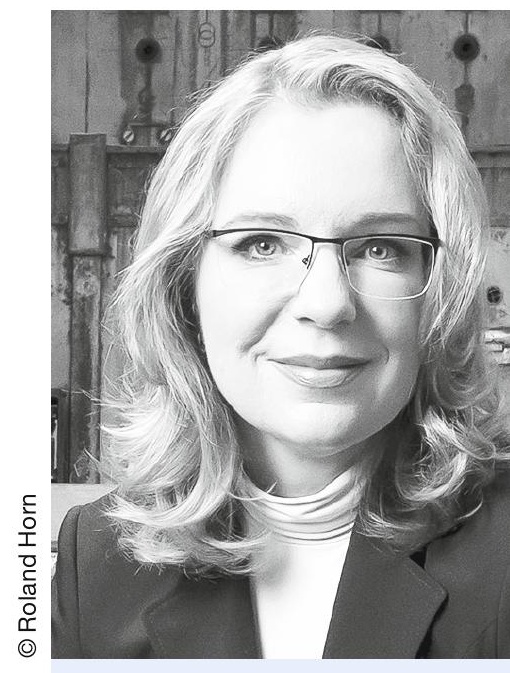

Claudia Kemfert ist Leiterin der Abteilung Energie, Verkehr, Umwelt im Deutschen Institut für Wirtschaftsforschung (DIW Berlin) und Professorin für Energieökonomie und Nachhaltigkeit an der Hertie School of Governance Berlin. 
Genau dieser Eindruck soll erweckt werden. Tatsächlich stecken aber wohl auch ganz andere politische Interessen hinter dieser Attacke, aber auch hinter so manch anderem juristischen Winkelzug: Hinter den vermeintlichen Naturschützern stecken oftmals Leugner des Klimawandels und Gegner der Energiewende. Sie wollen nicht die Wirtschaft per se blockieren, sondern nur die ökologische Wirtschaft der Zukunft, um die fossile Wirtschaft der Vergangenheit möglichst lange künstlich am Leben zu halten.

Diese Blockierer nutzen dieselben Strategien, wie wir sie inzwischen in vielen Bereichen erleben. Sie sagen mit rechtsstaatlichen Mitteln und unter Berufung auf Meinungsfreiheit oder jetzt eben Naturschutz der Demokratie den Kampf an. Blockade um der Blockade willen. Streit um des Streites willen. Denn je länger sich die ökologische Transformation hinzieht, desto länger klingeln die fossilen Kassen. Je mehr sich die demokratischen Kräfte aufreiben, desto leichter wird es für anti-demokratische Kräfte, sich durchzusetzen.

Deswegen ist es ganz sicher nicht angebracht, die Klagemöglichkeiten für Naturschutzverbände einzuschränken oder gar abzuschaffen. Es wäre auch fatal, die Meinungsfreiheit einzuschränken, um die wachsende Hatespeech-Kultur zu beenden. Wir würden die Demokratie mit dem Bade ausschütten.

Sicher sollten wir über bundeseinheitliche und vereinfachte Genehmigungsverfahren nachdenken, damit Deutschland möglichst viele solcher zukunftsfähigen Investitionen anzieht. Aber der angeblich so überbordenden und selbstgefälligen Bürokratie in Brandenburg ist kein Vorwurf zu machen. Das mit Industrie bislang nicht gerade gesegnete Bundesland reagiert erfreulich schnell und effektiv. Glücklicherweise wurde die Klage abgewiesen. Die Justiz muss generell erkennen, wenn sie von Interessengruppen, die leicht durchschaubar unter falscher Flagge segeln, instrumentalisiert werden. Das darf nicht sein. Mit juristischen Taschenspielertricks sollten weder Politik noch Wirtschaft in unserem Land manipuliert werden können. Das würde zugleich das Vertrauen in den Rechtsstaat zerstören. Genau das wollen die Leugner des Klimawandels ja erreichen.

Dieses Mal hatten sie keinen Erfolg. Gut so. Der Wirtschaftsstandort Deutschland hat keinen Schaden genommen. Im Gegenteil: Es zeigt sich, dass die Umweltstandards in Deutschland hoch sind, das Rechtssystem funktioniert und gleichzeitig Klimaschutzbelange hoch im Kurs stehen. Nun hat es Brandenburg selbst in der Hand, weltweit führend zu werden in puncto echtem Klimaschutz.

Tesla und Brandenburg sollten sich darauf einigen, dass für den Autobau auf erneuerbare Energie gesetzt wird und nicht auf fossile Energie aus Gas - nicht nur um des Klimas Willen, sondern auch wegen der Signalwirkung: Es reicht nicht, Autos mit Ökostrom zu fahren, sie müssen auch mit erneuerbaren Energien gebaut werden. Nur dann wird die Giga-Factory zum wirklich klimaneutralen Vorzeigewerk der Zukunft.

Wenn Brandenburg außerdem zügig aus der Kohle aus- und in eine Vollversorgung mit erneuerbare Energien einsteigt, könnte aus der oft verspotteten „Berliner Provinz“" in wenigen Jahren Deutschlands stärkster Magnet für Zukunftsinvestitionen werden. Dazu gehört - wenn die Gegner nicht auch das trickreich sabotieren - allerdings auch ein deutlicher Ausbau der Windenergie. Teslas Paukenschlag kann der Auftakt für eine wirklich große Sinfonie werden: Anstelle des rückwärtsgewandten Polit-Getöses

Claudia Kemfert

DIW Berlin, Hertie School of Governance sekretariat-evu@diw.de erklängen dann bald neuartige Brandenburger Konzerte: Ökologie und Ökonomie harmonisch im Zusammenspiel. Ein Sieg von Demokratie, Rechtsstaat und verantwortungsvoller Wirtschaft. Eine Ode an die Freude. 